\title{
Apprentissage de l'universalisme citoyen en France
}

Premiers résultats d'une enquête à l'école primaire

Learning to be a universal citizen in France. Initial findings of a study in primary schools

Aprendizaje del universalismo ciudadano en Francia. Primeros resultados de una encuesta en la escuela primaria

\section{Géraldine Bozec et Sophie Duchesne}

\section{OpenEdition Journals}

Édition électronique

URL : https://journals.openedition.org/ries/167

DOI : 10.4000/ries. 167

ISSN : 2261-4265

Éditeur

France Education international

Édition imprimée

Date de publication : 1 avril 2007

Pagination : 95-104

ISBN : 978-2-85420-569-5

ISSN : $1254-4590$

\section{Référence électronique}

Géraldine Bozec et Sophie Duchesne, «Apprentissage de l'universalisme citoyen en France », Revue internationale d'éducation de Sèvres [En ligne], 44 | avril 2007, mis en ligne le 22 juin 2011, consulté le 05 juillet 2021. URL : http://journals.openedition.org/ries/167 ; DOI : https://doi.org/10.4000/ries. 167 


\title{
Apprentissage de l'universalisme citoyen en France
}

\section{Premiers résultats d'une enquête à l'école primaire}

\author{
Géraldine Bozec \\ Sophie Duchesne
}

La conception française de la citoyenneté a été forgée dans un contexte de lutte entre républicains et catholiques ${ }^{1}$ et de conquête de la démocratie ${ }^{2}$. Elle se caractérise par une discontinuité volontaire entre la personne dotée d'intérêts et de préférences liés à ses diverses appartenances (sociales, religieuse, territoriales, etc.) et le citoyen, être abstrait doué d'une raison capable de le dissocier de ses appartenances pour le tourner vers la volonté générale et le fondre dans la communauté politique, nationale et républicaine. C'est à l'École qu'est confiée la lourde tâche de "faire» des citoyens : éduquer leur raison et leur transmettre connaissances et attitudes qui leur permettent de dépasser les solidarités induites par leurs appartenances primaires; faire d'eux des êtres égaux et semblables dans leur effort pour accéder à des valeurs et des justifications morales et politiques universelles incarnées dans la nation française, héritière de la Révolution. Historiquement, la laïcité d'une part, et la centralisation et l'homogénéisation du territoire national de l'autre, permettent toutes deux de rejeter dans l'espace privé deux éléments forts de différenciation entre les citoyens, le religieux et le local, tandis que l'avènement du suffrage universel masculin, qui supprime progressivement les exigences en matière de patrimoine, de ressources et de statut professionnel dans la définition de l'électeur, produit une forme de neutralisation des différences sociales qu'on a résumée avec la formule: un homme, une voix ${ }^{3}$. Mais si le modèle de citoyenneté de la III $^{\mathrm{e}}$ République promeut des individus-citoyens capables de dépasser leurs intérêts et leurs attachements particuliers, il n'en fait pas pour autant des êtres purement indépendants : il les inscrit en effet dans un horizon de solidarité plus vaste, représenté en premier lieu par la nation. C'est bien le sens de «l'idéal collectif intermédiaire q qui caractérise, selon Yves Déloye, l'éducation civique scolaire de cette période : fondée sur la volonté individuelle, la citoyenneté qu'elle cherche à apprendre n'en vise pas

1. Déloye (Y.), Ecole et Citoyenneté. L'individualisme républicain de Jules Ferry à Vichy : Controverses, Paris, Presses de la FNSP, 1994.

2. Rosanvallon (P.), Le sacre du citoyen. Histoire du suffrage universel, Paris, Gallimard, 1992.

3. Rosanvallon (P.), Le peuple introuvable. Histoire de la représentation démocratique en France, Paris, Gallimard, 1998. 
moins une finalité communautaire, l'attachement des futurs citoyens, tout à la fois affectif et rationnel, à la nation ${ }^{4}$.

Le rôle actif de l'école publique, et tout particulièrement de l'école primaire, dans l'apprentissage de la citoyenneté est un trait fondamental de la République française. Il s'est perpétué depuis la III ${ }^{\mathrm{e}}$ République, et n'a connu qu'un bref moment de mise en sommeil dans les deux décennies qui ont précédé sa remise à l'honneur par Jean-Pierre Chevènement en 1985 quand l'éducation civique est réintroduite comme un enseignement à part entière, avec programmes et horaires, notamment à l'école primaire. Au cours des années 1990 et 2000, les différents gouvernements, de droite comme de gauche, ont prolongé cette action de réhabilitation. Les programmes pour l'école primaire ont été modifiés en 1995, puis en 2002. Les textes officiels actuels font de l'éducation civique un "domaine transversal», nourri, en cycle 3, par l'ensemble des disciplines (une heure par semaine) et par les débats en classe sur les problèmes de la vie collective (une demi-heure hebdomadaire). En 2007, l'éducation civique est officiellement le deuxième pilier des programmes de l'école primaire, après la maîtrise de la langue ${ }^{5}$.

Cet article rend compte des premiers résultats d'une enquête sur l'éducation civique en France aujourd'hui à l'école primaire ${ }^{6}$. Il vise à faire le point sur les contenus actuels de cet enseignement, à travers les programmes d'abord, mais aussi et surtout à travers la façon dont les instituteurs les interprètent et les mettent en pratique. Il montre que si la dimension individualiste de la citoyenneté se perpétue, voire se renforce aujourd'hui, la solidarité et le lien entre co-citoyens qui venaient la contrebalancer, autrefois portés par l'attachement à la nation, peinent à trouver de nouveaux fondements.

\section{UNIVERSALISME ET RESPECT DES DIFFÉRENCES : UN COMBAT INÉGAL}

«Respecter la différence, dans la mesure où cela ne porte pas atteinte aux valeurs universelles des droits de l'homme " ${ }^{7}$ : voilà ce que l'éducation civique vise aujourd'hui à apprendre aux enfants. Si les programmes scolaires évoquent les différences, c'est pour mieux rappeler, immédiatement, la norme

\footnotetext{
4. Déloye (Y.) : op. cit., en particulier p. 106 et suivantes. L'idéal collectif qui anime l'éducation civique rejette à la fois l'individualisme exacerbé aboutissant au règne des intérêts privés et à l'atomisation de la société, et la fusion dans une communauté fondée sur la ressemblance entre individus et leur origine commune.

5. Ministère de l'éducation nationale (MEN), Qu'apprend-on à l'école élémentaire? 2006-2007 (http://www.cndp. fr/ecole/) p. 10.

6. Cette enquête s'inscrit dans le cadre d'une recherche doctorale (thèse de G. Bozec dirigée par S. Duchesne) qui porte sur le type de communauté politique mise en scène à l'école primaire, à travers les débats éducatifs d'une part, les représentations et les pratiques des instituteurs d'autre part. Le matériau plus particulièrement mobilisé est constitué d'entretiens auprès d'un échantillon diversifié d'enseignants (en termes d'âge, de sexe, de type de carrière, d'origines sociales, d'opinions politiques, etc.). Pour une présentation plus exhaustive de la recherche, voir notamment: (http://www.cevipof.msh-paris.fr/rencontres/smnr/sociopo_index.htm).
}

7. MEN, op. cit., p. 176. 
qui prévaut sur la diversité : l'égalité des droits. Cette hiérarchie entre égalité et différence se retrouve dans le discours de la majorité des instituteurs interrogés. Expressions fréquentes dans les entretiens, le «respect des autres», le «vivre ensemble» sont souvent rapportées à la question de l'égalité entre enfants. Les élèves doivent avant tout apprendre à l'école qu'ils sont égaux et appartiennent au même groupe humain, quelles que soient leurs particularités individuelles ou collectives (apparence physique, caractère, appartenance ethnique, sexe, milieu social...). C'est en ceci notamment que l'éducation civique est bien plus qu'une discipline scolaire, conformément aux instructions officielles. À côté des savoirs institutionnels ou historiques, maints moments de la vie scolaire - un incident survenu entre deux élèves, le rejet d'un enfant du groupe, l'étude d'un texte littéraire ou d'une période de l'histoire - fournissent des occasions de rappeler aux enfants leur égalité fondamentale en tant qu'être humains. Beaucoup d'enseignants travaillent avec leurs élèves sur des thèmes ayant trait aux droits de l'homme et aux discriminations: le racisme et le sexisme, en particulier, sont des sujets souvent abordés dans les classes. Mais ces séances sont avant tout l'occasion de rappeler la norme égalitaire et les interdits qui l'accompagnent, plus que de réfléchir aux inégalités entre les groupes qui sont à l'œuvre dans la société.

Dans le discours de bien des instituteurs, en particulier de ceux qui travaillent dans des établissements multiethniques, la crainte de la création de groupes distincts au sein de l'école est très perceptible. La différence ethnique et religieuse est perçue comme présentant toujours le risque de créer des groupes au sein de la communauté des enfants et, entre ces groupes, des rapports de domination et des conflits. Une institutrice dénonce par exemple le regroupement d'enfants noirs dans la cour de récréation dès qu'un enfant noir est interpellé par les enseignants suite à un conflit entre élèves. Il est intéressant de remarquer que, bien qu'elle soit consciente que cette attitude est sans doute la conséquence d'un sentiment d'injustice ressenti par ces élèves, elle n'essaie pas de réfléchir avec eux sur la réalité de cette injustice. En accord avec l'équipe pédagogique de son école, elle se contente d'interdire ce type de manifestation de solidarité. La «couleur de peau » ou bien la religion sont immédiatement refoulées et traitées comme des critères d'identification illégitimes, opposées aux valeurs incarnées par «l'école publique».

La crainte des revendications identitaires porte clairement sur les minorités, qu'elles soient ethniques (enfants issus d'Afrique du Nord ou d'Afrique noire) ou religieuses (l'islam). En cela, la perception de beaucoup d'enseignants recoupe les positions de leurs ministres de tutelle successifs, qui présentent le communautarisme comme l'un des dangers principaux auxquels sont confrontés l'école et plus largement le modèle français de citoyenneté ${ }^{8}$.

8. Voir le Guide républicain : l'idée républicaine aujourd'hui publié par le ministère de l'éducation nationale (2004) et notamment la préface de François Fillon et l'introduction de Luc Ferry, ainsi que la circulaire envoyée par François Bayrou en 1994 aux autorités académiques et aux directeurs d'établissements scolaires sur le port de signes religieux à l'école. 
Certes, tous les enseignants n'ont pas la même attitude face aux possibles répercussions de la diversité ethnique et religieuse dans leur classe. Dans le discours de certains, la peur des divisions communautaires et la stigmatisation des attitudes des minorités ethniques et religieuses est effectivement ce qui domine. Dans d'autres entretiens, l'attitude des enseignants est plus ambivalente car l'on trouve aussi une volonté de comprendre le rejet subi par ces groupes minoritaires et, à certaines occasions, de valoriser leurs cultures d'origine. Enfin, une minorité d'enseignants se distingue des autres par un discours plus construit et bien plus engagé sur la mise en valeur de la diversité culturelle.

Quoi qu'il en soit, la mise en avant d'une France plurielle n'apparaît que rarement comme une dimension centrale dans les commentaires des enseignants. Ceux-ci trouvent d'ailleurs peu d'incitations en ce sens dans les programmes scolaires, qui restent largement silencieux sur cette question, en dehors de courts passages dans la section consacrée aux langues étrangères et régionales ${ }^{9}$. De fait, les instituteurs insistent beaucoup plus sur l'ouverture à d'autres cultures que sur la mise en avant d'une France elle-même multiculturelle.

La norme égalitaire ne s'applique pas seulement aux différences ethniques et religieuses : elle entraîne aussi le plus souvent l'occultation des différences sociales. Ainsi, une institutrice et directrice d'école dans un village du sud de la France se félicite en entretien, alors qu'elle commente les rédactions écrites par ses élèves, que les enfants évitent de faire référence à leur richesse pour se définir ${ }^{10}$. Elle juge préférable que les différences sociales soient ignorées des élèves et, ce qui est plus notable, par l'école elle-même, considérant qu'à l'école " on ne doit pas faire la différence entre les riches et les pauvres, on devrait être tous pareils». La volonté d'affirmer le statut égal des élèves dans l'institution scolaire déborde le respect de la différence : il ne s'agit plus seulement de rappeler que les individus sont à la fois différents et égaux, mais bien d'exclure la différence (ici, sociale) de la sphère légitime de l'école.

En fait, le rapport des enseignants aux appartenances sociales des enfants et plus largement à leurs contextes familiaux est bien souvent empreint d'ambivalences. Le fait même que certains veuillent les ignorer peut paraître surprenant dans la mesure où ces instituteurs semblent en même temps très conscients que l'environnement familial et social de l'enfant pèse sur ses comportements et ses résultats scolaires. Mais la norme d'égalité de traitement des enfants joue suffisamment pour que certains instituteurs choisissent (ou affichent) de «ne pas en savoir plus».

9. Ces passages insistent sur l'enrichissement lié à la diversité linguistique et culturelle, y compris sur le sol français (voir notamment MEN : Qu'apprend-on..., op. cit., pp. 124 et 199).

10. L'entretien avec les enseignants était en effet constitué de deux parties. Dans un premier temps, ils devaient réagir aux textes qui avaient été écrits en classe par leurs élèves à partir d'une consigne ("dire qui ils sont») et d'une liste d'exemples commentée par l'enseignant. Un des items proposés dans cette liste portait sur le niveau de richesse : «chez nous, on est plutôt riche, on n'a pas beaucoup d'argent, on est dans la moyenne». Dans un second temps, les enseignants répondaient à une série de questions sur leurs pratiques et leurs représentations (concernant notamment l'éducation civique). 
Au-delà des différences sociales, le rapport général que l'école entretient avec la société est ambivalent. D’un côté, conformément aux instructions, l'école est considérée comme un échantillon de la société, dans lequel les enfants doivent faire l'expérience des règles, des principes et des lois de la République $^{11}$. C'est le cas lorsqu'il s'agit de pénaliser des discriminations, par exemple en supprimant les jeux de football lorsque les garçons refusent de jouer avec les filles ${ }^{12}$. Mais l'école est aussi souvent, pour les enseignants, un lieu à part, une "forteresse» dit une institutrice, dans laquelle ils traitent les enfants - les futurs citoyens - sur un mode idéal, à rebours de ce qui se passe dans la vie réelle. En cela, l'école porte doublement l'empreinte de la France républicaine qu'elle représente en tant que lieu de formation des futurs citoyens : espace exemplaire de mise en œuvre de la légalité républicaine, elle rend manifeste la persistance d'une conception abstraite de l'individu citoyen - ici de l'enfant futur citoyen. La collectivité que les enseignants cherchent à construire dans l'espace scolaire trouve son fondement dans la figure de l'individu détaché de ses appartenances primaires spécifiques, et sa finalité dans l'universalisme de l'appartenance de tous à la même communauté humaine.

\section{L'APPARTENANCE NATIONALE ENTRE NON-DIT ET CONTESTATION}

Un autre élément important de l'éducation civique aujourd'hui, présent également derrière l'idée de «respect des autres», consiste à apprendre aux enfants les bases d'une civilité minimale dans leurs rapports mutuels. Cet apprentissage se fait aussi au quotidien. Cela passe essentiellement par le refus des insultes, le recours «à la parole plutôt qu'à la violence» en cas de différends ${ }^{13}$ et, plus généralement, par l'impératif de la prise en compte de l'autre.

On retrouve là un des éléments de l'éducation civique scolaire de la III ${ }^{\mathrm{e}}$ République, qui promouvait aussi le dépassement de l'individualisme par le souci des autres et «le gouvernement de soi» ${ }^{14}$. Cependant, cela dessine aujourd'hui une morale civique particulière bien peu cohésive : elle est avant tout assise sur une coexistence pacifique entre les enfants et fondée sur les efforts faits pour respecter l'autre, ne pas le gêner et le considérer comme son semblable. Par comparaison, sous la III $^{\mathrm{e}}$ République, l'idée de solidarité était

11. MEN, Qu'apprend-on..., op. cit., p. 177. Il est ainsi fait référence à l'école comme «petite société » et à son « règlement intérieur » comme «mettant en application» les «lois républicaines».

12. Une partie de l'entretien était consacrée à la gestion, par l'enseignant, des relations et des conflits entre élèves. Différents scénarios mettant en scène des situations banales, souvent observées dans les écoles, étaient proposées (dont un, précisément, sur l'exclusion des filles des jeux de football pendant la récréation). Il était demandé à l'enseignant de dire comment il réagirait dans de tels cas.

13. Maroussia Raveaud souligne aussi, dans son enquête comparative sur l'éducation en France et en Angleterre, l'importance de la discussion dans le traitement des différents entre enfants. Voir : Raveaud (M.), De l'enfant au citoyen. La construction de la citoyenneté à l'école en France et en Angleterre, Paris, PUF, 2006, pp. 100-109.

14. Déloye, op. cit., en particulier pp. 88-96. 
très développée dans l'enseignement civique et moral. Jean Baubérot en trouve une empreinte forte dans les cahiers d'élèves qu'il étudie : la solidarité s'appuyait là sur la dette contractée par l'individu à l'égard de la société qui lui apportait ses bienfaits. La nation constituait le lieu privilégié de cette solidarité entre individus. Les enfants découvraient la "grande patrie», dont la grandeur devait autant aux souffrances de leurs ancêtres qu'au travail et à l'engagement civique des générations présentes. Du passé et du présent découlait leur avenir : il leur reviendrait de continuer à défendre cet héritage et de participer à leur tour à la grandeur de la nation ${ }^{15}$. Aujourd'hui, la référence à la solidarité est singulièrement faible, sinon totalement absente, du discours des instituteurs. Est-ce parce que, précisément, la légitimité du cadre national s'est affaiblie? En effet, que reste-t-il de l'apprentissage de la nation à l'école, dans ce contexte de crainte des manifestations identitaires des minorités, d'intégration européenne croissante et de débats sur la possibilité d'une citoyenneté post-nationale?

Il est de fait très difficile de repérer, dans le discours des instituteurs, une quelconque volonté de développer, chez les enfants, un attachement à la nation française. À première vue, ce qu'il s'agit d'apprendre aux élèves, c'est la République, ce qu'elle a apporté et continue d'apporter aujourd'hui. Sur ce point, les enseignants s'inscrivent en continuité par rapport aux programmes scolaires, qui développent avant tout l'image d'une nation républicaine. "Être citoyen en France» - un des titres de chapitre du programme d'éducation civique en cycle 3 - c'est comprendre qu'on «appartient à une nation démocratique», connaître la Déclaration des droits de l'homme et du citoyen et savoir ce qui constitue la spécificité d'une République (par opposition à la monarchie). La plupart des enseignants rencontrés dans l'enquête insistent ainsi, en éducation civique et en histoire, sur la Révolution française, la Déclaration des droits de l'homme et du citoyen, l'installation de la République et les changements fondamentaux dont a été porteur ce premier acte de l'histoire républicaine. L'histoire qu'ils enseignent est, parfois explicitement, au service d'un message civique, quitte à ne pas toujours refléter la complexité et la discontinuité historiques, une sorte de linéarité étant établie entre l'événement fondateur qu'est la Révolution française et le régime actuel. Dans ce discours scolaire, l'identité nationale apparaît finalement comme peu spécifiée : certes, les enfants apprennent l'histoire de la République en France mais, ce faisant, ils apprennent aussi l'importance des droits universels. L'appartenance à la nation n'est pas valorisée en tant que telle : c'est surtout à la République en ce qu'elle est porteuse de droits universels que l'attachement des enfants est sollicité.

15. Baubérot (J.), La morale laïque contre l'ordre moral, Paris : Editions du Seuil, 1997. D'autres auteurs ont souligné le rôle de l'école dans l'apprentissage du sentiment national, en particulier Weber (E.), La fin des terroirs. La modernisation de la France rurale, 1870-1914. Paris, Ed. Recherches, 1983. Pour une étude des manuels scolaires en ce sens, voir Déloye, École et citoyenneté..., op. cit., notamment pp. 96-116. 
Cependant, quelques entretiens laissent percevoir, dès lors qu'il est question d'enfants d'origine étrangère, un autre rapport entre identité nationale et citoyenneté. Dans l'esprit de quelques enseignants travaillant notamment dans des écoles multiethniques, l'existence d'un sentiment d'appartenance à la nation française semble un préalable nécessaire à la citoyenneté. Ainsi, une institutrice réagit vivement au texte d'un enfant qui se dit de nationalité tunisienne bien qu'il soit né en France. Elle explique alors mieux comprendre l'attitude de certains élèves en classe d'éducation civique : «ce que je vois, c'est quand on leur apprend des règles et des lois de la nation française, ils trouvent toujours beaucoup d'arguments pour s'en démarquer et pour dire "oui mais à tel endroit, c'est comme ça, là c'est comme ça et puis moi je préfère faire comme ça, c'est pas normal, ça se fait pas, c'est pas juste”. Et je comprends mieux maintenant qu'effectivement, ils n'ont pas ce sentiment d'appartenance à la nation française.» Le lien est fait ici entre l'acceptation des règles et le sentiment d'appartenance à la nation qui les édicte, dont l'absence semble étonner, voire choquer certains enseignants. À l'inverse, quelques autres enseignants vont s'insurger contre toute consigne visant à favoriser l'appartenance à la France dans les repères voire l'affection des enfants, les jugeant "patriotiques ».

En définitive, c'est bien la question de la place des enfants issus de l'immigration qui semble le plus mettre en tension la conception universaliste de la citoyenneté, en faisant ressortir ses limites et en révélant, dans le même mouvement, des divergences entre les enseignants sur la diversité culturelle et le rapport au national. Certains, pour qui le sentiment d'appartenance nationale est une nécessité, ne l'explicitent pas et semblent tenir pour acquis (jusqu'à preuve du contraire) son existence; tandis que d'autres, qui se fondent sur l'histoire du $\mathrm{XX}^{\mathrm{e}}$ siècle pour se méfier du caractère exclusif de l'attachement national, trouvent les mots pour le dénoncer. Ainsi semble s'estomper ce qui servait de cadre privilégié à la solidarité des citoyens sous la III ${ }^{\mathrm{e}}$ République, la nation.

On pourrait faire l'hypothèse que la discrétion des programmes récents et de la majorité des instituteurs sur l'apprentissage d'un sentiment d'appartenance à la France est liée à un déplacement vers d'autres niveaux de réalisation de la citoyenneté, et tout particulièrement, du fait des développements institutionnels en ce sens, le niveau européen. Dans les programmes d'éducation civique, l'Europe fait partie d'un chapitre qui traite plus globalement de l'ouverture au monde. Sont notamment évoqués, à côté de l'Europe, la francophonie et certains problèmes mondiaux (les inégalités entre pays du monde, la question des droits de l'enfant, l'enjeu environnemental). L'Union européenne n'a donc pas véritablement de statut spécifique dans les programmes. En outre, elle n'est pas évoquée en termes de citoyenneté, comme nouvel espace d'identification et d'action politiques. Est plutôt mise en avant la découverte des autres pays européens à travers les langues étrangères, la géographie ou encore les 
correspondances scolaires ${ }^{16}$. L'absence de spécificité de l'Europe et l'accent mis sur sa diversité interne se retrouvent dans les déclarations des instituteurs. Certains enseignants, qui affirment leur attachement personnel à l'Europe, travaillent beaucoup sur ce thème. Mais de façon générale, les enseignants ne présentent pratiquement jamais l'Union européenne comme un nouveau lieu de citoyenneté. Les référents comme l'Europe, mais aussi le monde sont conçus par les instituteurs comme des univers collectifs trop abstraits et trop éloignés des enfants, qui sont avant tout centrés sur leur environnement immédiat. La seule façon de redonner une dimension concrète à ces univers plus larges est alors d'évoquer ce qui constitue leur spécificité : la diversité des pays et des cultures en leur sein. Concernant l'Europe, le travail scolaire le plus courant est ainsi de faire réaliser aux élèves une recherche sur différents pays européens, présentant notamment la langue, la capitale, le nombre d'habitants et le drapeau national du pays.

Être «citoyen du monde» ne constitue pas non plus un élément important. Certes, les enfants étudient très souvent les droits de l'homme et les droits de l'enfant et peuvent ainsi percevoir leur dimension universelle. Mais ce discours humaniste trouve peu d'écho en termes d'action civique : l'accent n'est pas mis sur l'idée d'appartenance à l'humanité comme porteuse de responsabilités et de solidarité entre les individus-citoyens.

L'absence des appartenances civiques européenne et mondiale dans le discours et les pratiques des enseignants n'est d'ailleurs pas étonnante en soi, si l'on considère qu'elles restent encore à inventer ${ }^{17}$. Leurs ressorts possibles font d'ailleurs débat dans la communauté savante. On notera par ailleurs que l'Europe et le monde apparaissent, par contraste avec l'école et la France qu'elle représente, comme des espaces légitimes pour parler de la diversité et des différences.

\section{UNE ÉDUCATION À LA CITOYENNETÉ SANS POLITIQUE}

Si la solidarité entre les futurs citoyens ne repose plus sur la nation et ne se nourrit pas d'une appartenance supranationale, est-ce dans la conscience d'une capacité à agir ensemble pour influer sur l'avenir que l'école entend amorcer le ressort de la citoyenneté? Apparemment non. Dans les discours des instituteurs, le rapport de l'individu au pouvoir en tant que citoyen est une dimension bien peu présente. Dans l'enquête, les enseignants qui y font référence spontanément comme un des thèmes de l'éducation civique sont l'exception :

16. MEN, Qu'apprend-on, op. cit., p. 178.

17. Voir Jamet (C.), Lenoir (Y.), Xypas (C.), École et citoyenneté : un défi multiculturel, Paris, Armand Colin, 2006. Les différents auteurs insistent sur l'impératif, en même temps que la difficulté, de faire vivre une citoyenneté européenne par et dans l'école. 
apprendre la citoyenneté est pour eux faire comprendre que l'on «peut agir sur la société». Globalement cependant, cette idée d'un pouvoir d'action des individus dans la vie collective et politique est rarement évoquée. Si «l'esprit critique» est très souvent mentionné comme une des attitudes clefs qu'il s'agit de favoriser chez les élèves à travers l'éducation civique, il faut noter que cela vise surtout le détachement de l'enfant par rapport aux préjugés familiaux, et bien moins la vigilance des citoyens dans la vie sociale et l'engagement politique. La participation politique n'est pratiquement pas évoquée par les enseignants, en dehors de sa forme la plus légitime et la plus traditionnelle : le vote.

Dans une partie des écoles de l'enquête, il existe des conseils d'enfants qui tentent de mimer, à l'échelon scolaire, les pratiques démocratiques : les délégués de chaque classe y discutent, en présence d'un adulte ou de plusieurs, des problèmes de la vie collective à l'école. Conformément aux programmes, certains enseignants organisent aussi régulièrement des débats sur la vie collective au niveau de la classe. Ces procédures de participation sont cependant très limitées. D’abord, elles ne concernent qu'une minorité d'écoles et de classes. De plus, elles sont conçues par les enseignants comme des lieux d'expression des élèves plus que comme des modes de participation au pouvoir de décision dans l'école. Elles sont même parfois l'occasion pour les adultes de rappeler les règles de la vie scolaire, voire de traiter les problèmes liés à la discipline.

Ces procédures ne sont donc pas nécessairement l'indice d'une pédagogie participative de l'éducation à la citoyenneté. Plus généralement, la politique elle-même est exclue de cet enseignement ${ }^{18}$. Les enjeux sociaux discutés dans l'actualité sont peu présents dans les discussions en classe. Les forces politiques et sociales (partis, syndicats, etc.) ne sont jamais évoquées en cours d'éducation civique. La politique est donc, le plus souvent, soigneusement évitée. La norme de neutralité scolaire peut rendre compte de ce phénomène, mais seulement en partie : en théorie, rien n'empêche l'enseignant d'évoquer, sans prendre parti, les débats politiques du moment et les acteurs qui les animent. Il semble, là encore, que «l'exclusion de la politique ${ }^{19}$ renvoie à l'inquiétude, dont témoignent bien des enseignants, de la division de la collectivité des enfants. La politique n'est donc abordée qu'à travers la présentation brève du fonctionnement des institutions nationales (et plus rarement, locales),

18. Analysant différentes pensées pédagogiques, François Galichet met à jour trois modèles d'éducation à la citoyenneté : «mimétique», «analogique» et «réaliste». Les deux premiers modèles, dans leur logique même, conduisent à exclure la politique de l'école : le premier, parce qu'il considère l'enfant comme un mineur en politique, incapable d'accéder à la compréhension de la vie politique, le second parce qu'il fait de l'école une métaphore parfaite de la société démocratique et qu'il est dès lors impossible de réintroduire «la réalité » (la politique) «dans le modèle réduit». Concernant le second modèle, il est justement intéressant de noter que même si les principes démocratiques animant, au moins en partie, les conseils d'enfants étaient poussés jusqu'à leur terme, cela ne signifierait en rien une meilleure formation des enfants à la politique : si les procédures sont démocratiques, les enjeux politiques de la société réelle sont absents. Galichet (F.), L'éducation à la citoyenneté, Paris, Anthropos, 1998.

19. Percheron (A.). La socialisation politique, Paris, A. Colin 1993, ch. 1 et 3. 
conformément aux programmes. Une partie des enseignants déclarent cependant ne pas s'y plier, trouvant cela «fastidieux», «rasoir» ou «trop compliqué pour les enfants».

\section{À LA RECHERCHE DU LIEN CIVIQUE?}

Cette enquête montre une continuité certaine entre l'éducation civique aujourd'hui en France et ce qu'elle était à l'époque de sa fondation. On retrouve la même volonté de former des citoyens égaux, quitte à rejeter, hors de la sphère scolaire, la vie politique et sociale dont les inégalités et les conflits entre groupes sont la trame. Mais l'autre pôle central de l'enseignement civique sous la III ${ }^{\mathrm{e}}$ République, l'affirmation de la solidarité entre concitoyens, apparaît actuellement absent dans le discours et les pratiques des instituteurs. L'appartenance nationale, ferment privilégié de cette solidarité il y a un siècle, n'est pas relayée aujourd'hui par d'autres appartenances civiques, malgré la prégnance d'un discours humaniste chez les instituteurs. Si l'on ajoute à cela l'exclusion de la politique et des solidarités forgées dans les conflits sociaux et idéologiques, on peut craindre que cette éducation civique, dont on attend apparemment beaucoup, ne manque son but : n'omet-elle pas de donner aux futurs citoyens la clef de leur pouvoir? Car ni les programmes ni les pratiques des enseignants ne contribuent fortement à installer, entre les élèves, futurs citoyens, un lien civique. Or n'est-ce pas ce lien - qu'il soit nourri de l'héritage d'un passé commun ou du désir d'écrire ensemble le futur - qui confère aux citoyens la force d'agir ensemble et d'éprouver ce qui fait leur force : la puissance du nombre? 